\title{
ALORT: a transport layer protocol using adaptive loss recovery method for WSN
}

\author{
AYHAN KİRAZ ${ }^{1, *}$ and MURAT ÇAKIROĞLU ${ }^{2}$ \\ ${ }^{1}$ Department of Computer Engineering, Bozok University, Yozgat, Turkey \\ ${ }^{2}$ Department of Mechatronics Engineering, Sakarya University, Sakarya, Turkey \\ e-mail: ayhankiraz@bozok.edu.tr; muratc@sakarya.edu.tr
}

MS received 24 February 2016; revised 16 November 2016; accepted 20 December 2016

\begin{abstract}
Recently, critical projects were developed using wireless sensor network (WSN) such as medical and military projects. Performing reliable communication in WSN is extremely important for such projects. To be able to perform reliable communication, packet loss must be minimal. Packet loss, energy and latency are the most serious problems for transport protocols. In this paper, reliable, energy- and delay-sensitive transport layer protocol (ALORT) has been developed for WSN. The proposed protocol provides optimum reliability, optimum packet latency, minimum energy cost and high packet delivery ratio by changing loss recovery mechanism (LRM) according to channel error rates. ALORT algorithm is compared with PSFQ and DTC in terms of packet delivery ratio, end-to-end latency and energy cost by MIXIM framework in OMNET++. ALORT algorithm reduces energy cost and end-to-end latency and increases packet delivery ratio.
\end{abstract}

Keywords. WSN; transport protocol; reliability; sensor network; bit error rate.

\section{Introduction}

In recent years, there have been developments in processor, memory and radio frequency areas and have led to a variety of sensors to be integrated into devices that are capable of wireless communication. These technological developments have resulted in developing wireless sensor networks (WSNs) that observe a region remotely and undertake various multimedia communications. WSNs consist of small and portable wireless sensor devices. Areas of usage are widespread, such as in military, health care, ensuring the safety of a building and detecting forest fires [1]. Wireless sensors generally have two standard batteries, limited data storage/processing capacity and short-range communication over wireless environments.

WSNs are separated from other wireless networks according to their structural properties. For example, while network node density of WSN is extremely large compared to the others, data transmission rate is very low. Furthermore, the size of nodes in WSN is smaller and radio receiver/transmitter quality is much lower than other wireless networks [1]. Using low-quaslity receivers/transmitters in WSN leads to more packet loss. Packet loss may lead to very serious problems especially in critical fields such as military and medical. For this reason, the researchers emphasised a necessity for the usage of transport layer protocols for providing reliable communication

*For correspondence in critical applications where packet losses are significant [2]. Transport layer protocols generally guarantee reliable transmission of data packets from end to end by dividing large-size packets into small segments. In addition, protocols minimise packet loss by solving congestion problems. In the literature, there are various transport protocols that provide high reliability, low power consumption, low endto-end latency and high bandwidth designed for WSN $[1,3]$.

The proposed protocol (ALORT) is designed to achieve the best results in all environmental conditions. Two different LRMs are used for recovering lost packets. They are end-to-end and hop-by-hop loss recovery methods. Both LRMs have their advantages based on the environmental conditions. End-to-end LRM provides faster segment transmission and lower end-to-end packet latency values than hop-by-hop LRM in low error rates. But it provides slower transmission and higher end-to-end packet latency than hop-by-hop LRM in high error rates. In addition, hopby-hop LRM provides faster segment transmission and lower end-to-end packet latency than end-to-end LRM in high error rates. But it provides slower transmission and higher end-to-end packet latency than end-to-end LRM in low error rates.

The aim of this study is to design a protocol that has lower packet latency and faster transmission by using advantages of these two LRMs.

The properties and advantages of the ALORT are listed as follows: 
- Changing LRM in accordance with channel error rates,

- High packet delivery ratio and reliability,

- Low end-to-end packet latency,

- Low energy consumption.

ALORT was compared with DTC and PSFQ protocols and it was seen that ALORT is superior to the other protocols.

The rest of the paper is organised as follows. Section 2 presents a review of related work and loss recovery methods in transport protocols. Section 3 describes the proposed ALORT protocol in details. Section 4 presents the performance evaluation of the proposed protocol and section 5 presents the conclusions.

\section{Related work}

We now briefly summarise the well-known end-to-end and hop-by-hop transport layer protocols for WSN. For brevity, only a few protocols were observed, but an interested reader is referred to relevant survey papers.

\subsection{End-to-end transport protocols for WSNs}

Transport layer generally has two tasks in WSNs. The first of these is to detect and solve the network congestion problems and the second is to guarantee reliability of packets [4]. In spite of the general acceptance, some transport layer protocols such as STCP [5], ESRT [6], TRCCIT [7], CRRT [8], $\mathrm{RT}^{2}$ [9], ART [10], RCRT [11], Flush [12], PORT [13] support congestion and reliability control together, many of them consider only one task. For example, Fusion [14], CODA [15], CCF [16], PCCP [17], ARC [18], Siphon [19], Trickle [20], PHTCCP [21], SenTCP [22] only handle the congestion problem [23]. Reliable protocols are focused on providing reliability and are categorised in the literature based on many different aspects. In this study, reliability protocols were examined under three main categories: reliability aspect, reliability type and LRM.

\subsection{Hop-by-hop transport protocols for WSNs}

Providing reliability from sink to nodes is called downstream and from nodes to sink is called upstream protocols. Another category is based on the type of reliability protocols. In the literature, two different types of reliability are mentioned. The first one is the packet reliability and the other is event reliability. All segments must be transmitted successfully to provide packet reliability in transport protocols. Lost segments must be defined and must be recovered quickly. Successful transmission of all segments is not important in event-based reliability protocols. Another type of classification in reliability-based protocols is loss recovery methods. In the first method, lost segments are recovered by using hop-by-hop method and in the second method lost segments are recovered by using end-to-end method. Intermediate nodes are responsible for detecting and recovering lost segments in hop-by-hop method. Intermediate nodes that realise received wrong sequence number request lost segments from neighbour nodes with a NACK packet. After recovering loss segments, they can be sent to the next node. In order to recover loss segments from intermediate nodes, segments need to be buffered. In end-to-end LRM, only the target node is responsible for detecting loss segments. According to a segment timeout or unordered segment number, the target node generates a NACK packet. The target node requests missing segments from source node. The source node finds missing segments from buffer and transmits to the target node again.

\section{Protocol description}

Two different methods (hop by hop and end to end) are used for recovering lost packets [24]. In traditional end-toend method, target node requests missing segments from source and tries to recover them. In hop-by-hop method, intermediate nodes are responsible for recovering missing segments. When LRMs were compared, hop-by-hop method performs achieves results in unreliable connections. End-to-end method provides faster segment transmission and low end-to-end latency values in low error rates. Several simulations were performed in order to compare advantages/disadvantages of two methods and to understand which method is more efficient for a reliable transport layer. In simulations, source nodes sent 10 packets containing 50 segments to target nodes. Packet latency values in different channel error rates were recorded. In figure 1, average latency values are seen for both LRMs in seven hops network.

As seen in figure 1, end-to-end LRM ensures segment transmission with low packet latency in low error rates. When error rates increased, number of corrupted segments increase and recovery process of the loss segments may be more difficult. As a result, end-to-end latency values may be much more than hop-by-hop LRM. As understood in the graph, when error rate rises above $25 \%$ and end-to-end latency value rises above $3.3 \mathrm{~s}$, hop-by-hop LRM would be more advantageous instead of end-to-end LRM. Similar situations are valid for other hop counts such as 3, 5, 7 and 9. Therefore, using two LRMs (e2e and hbh) together enables better transport layer protocol design with delaysensitive instead of using one of them.

The proposed algorithm (ALORT) is a transport layer protocol that provides upstream reliability from source to the target node by using LRMs. ALORT optimises end-toend latency, low energy cost and packet delivery ratio values by changing LRMs according to channel error rates (figure 2). 


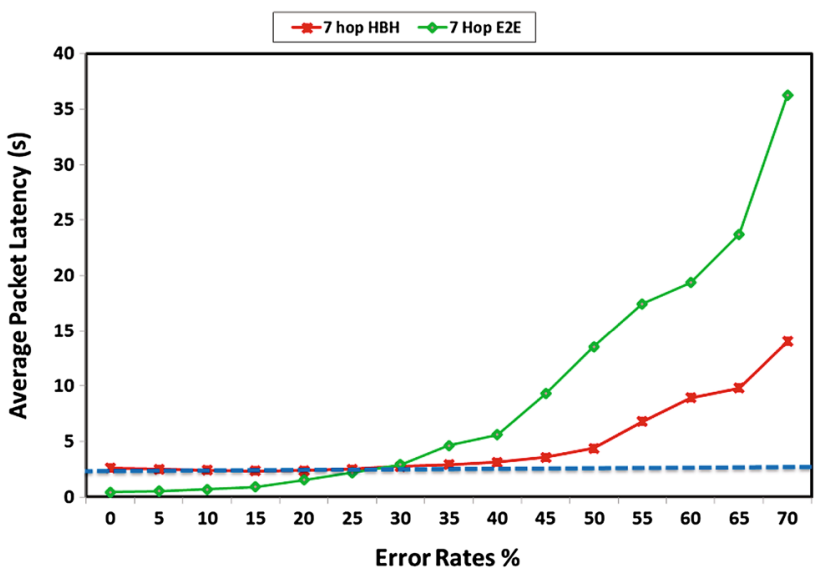

Figure 1. Determination of the transition parameters of end-toend and hop-by-hop methods.

\subsection{Determination of threshold values for detection of channel error conditions}

Although signal strength and quality can be measured by the physical layer parameters such as received signal strength intensity (RSSI) and line quality (LQ), detection of channel error rates is a rather difficult process in reality. There is no direct relationship between bit error rates and the aforementioned parameters. Therefore, getting information about transmission medium with end-to-end latency value is preferred instead of measuring channel error rates in ALORT. So a threshold mechanism is used to decide how LRM changes and in which latency value.

In threshold operation, source nodes store latency values of successfully delivered packets. They compare latency values and predetermined threshold values with specific intervals and decide which LRM will be used in the next packet sending process. In this study, several experiments were performed in different channel error rates to determine threshold values that the source nodes will use in MIXIM framework. Decider module of MIXIM framework was used to calculate signal-to-interference-plus-noise ratio (SINR) and to perform bit error rates. SINR value is used to determine bit errors of the Airframe [25]. In these experiments, grid topology with dimensions of $10 \times 10$ was used and each node sent packets by using two LRMs with different error rates. In the simulations, the source nodes sent 10 different packets consisting of 50 segments to the target node and recorded end-to-end latency values for both LRMs. In figure 3, the latency values obtained for different

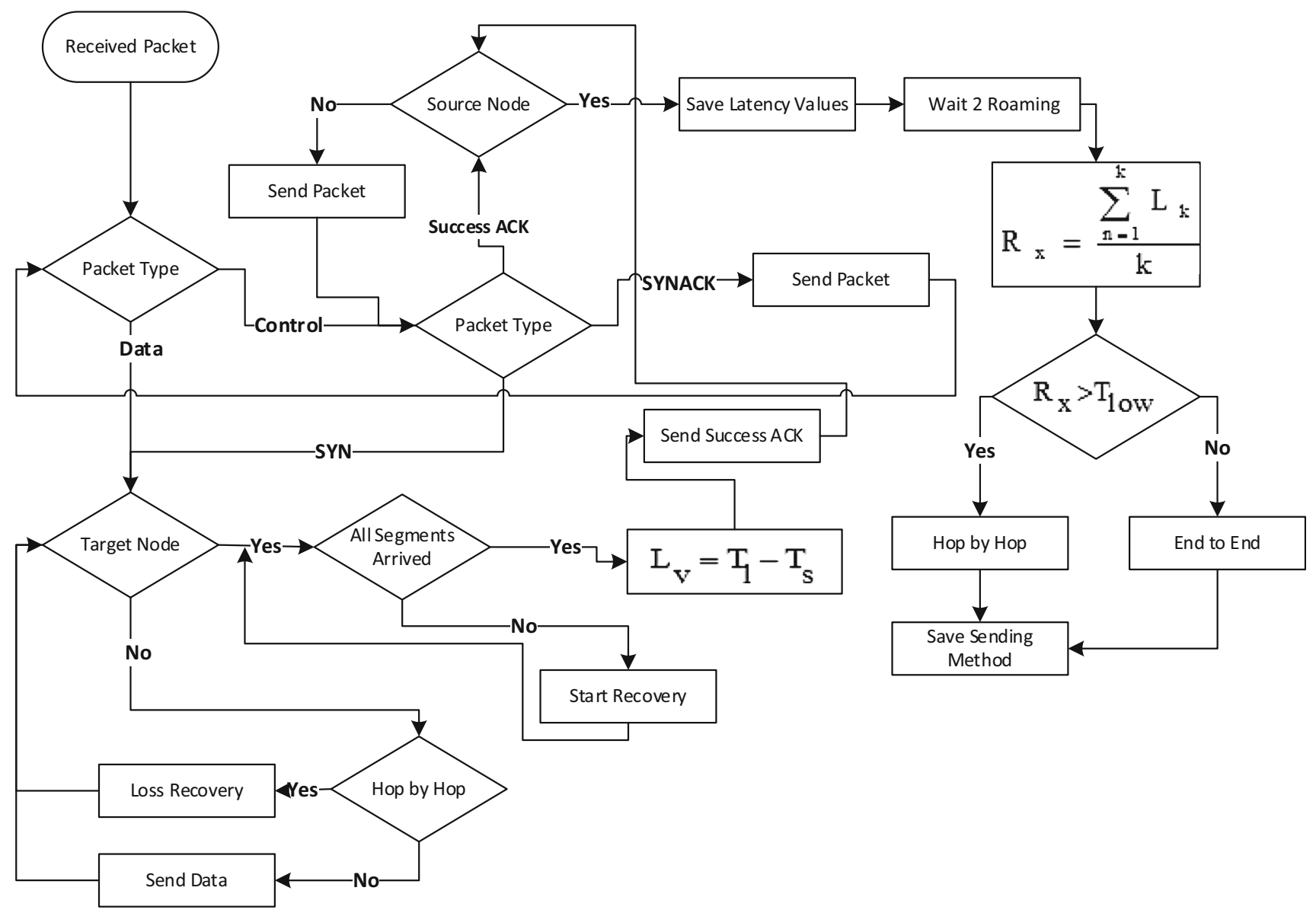

Figure 2. ALORT protocol diagram. 
hops can be seen. As seen in the graph, intersecting points of latency values of two methods are quite close. In other words, despite the different conditions, packet latency values of source nodes that switch from end-to-end to hopby-hop method are similar. Thus, global threshold value can be determined for all nodes on the network.

In this study, the graphs obtained were examined and determined with packet latency values. Normal distribution model of standard deviation was used to determine average packet latency value. Although it is simple, normal distribution provides effective modelling of many problems. According to the normal distribution, approximately $68 \%$ of values drawn from a normal distribution are within one standard deviation $(\sigma)$ away from the mean value $(\mu \pm \sigma$, where $\mu$ is the arithmetic mean); about $95 \%$ of the values lie within two standard deviations $(\mu \pm 2 \sigma)$; and about $99.7 \%$ are within three standard deviations $(\mu \pm 3 \sigma)$. It is called as the 68-95-99.7 rule. In this study, two threshold values are calculated for all nodes by using average and two standard deviations of latency values.

As seen in figure 4, lower threshold value called $T_{\text {low }}$ is used to switch from end-to-end to hop-by-hop method. This lower threshold value is calculated according to the latency distribution using the equation $\mu-2 \sigma$. Similarly, upper threshold value called $T_{\text {high }}$ is used to switch from hop-byhop to end-to-end method. $\mu+2 \sigma$ equation is used to calculate the $T_{\text {high }}$ upper threshold value.

When LRMs are changed adaptively in ALORT protocol, source nodes start to send packets with end-to-end LRM and store all latency values of sent packets. Then, source nodes compare previously calculated lower threshold latency values with an average latency of the round after performing a certain number of packet transmission called round. Thus, the source nodes decide whether switching method is necessary. If average latency in a round is more than $T_{\text {low }}$, next sending packet will be performed with hop-by-hop LRM. Source nodes compare $T_{\text {high }}$ threshold value and average latency value in that round after switching to hop-by-hop LRM. Thus, the switching process is controlled. In this case, if the average latency value in that round is less than $T_{\text {high }}$, the next sending packet will be performed with end-to-end LRM. In algorithm 1, pseudo code of the switching process is seen.
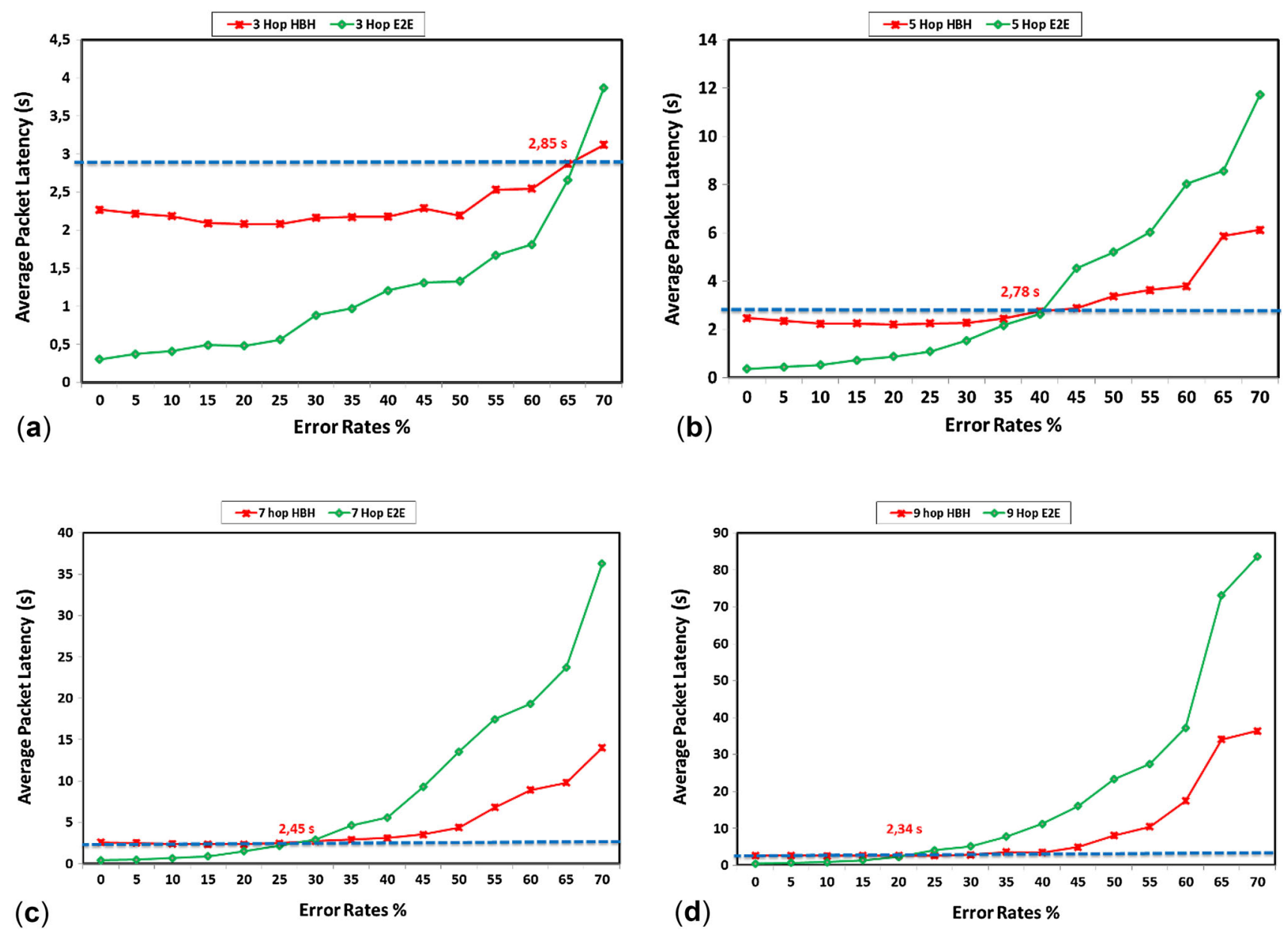

Figure 3. Hop-by-hop and end-to-end average packet latency values. (a) Three hops average packet latency value. (b) Five hops average packet latency value. (c) Seven hops average packet latency value. (d) Nine hops average packet latency value. 


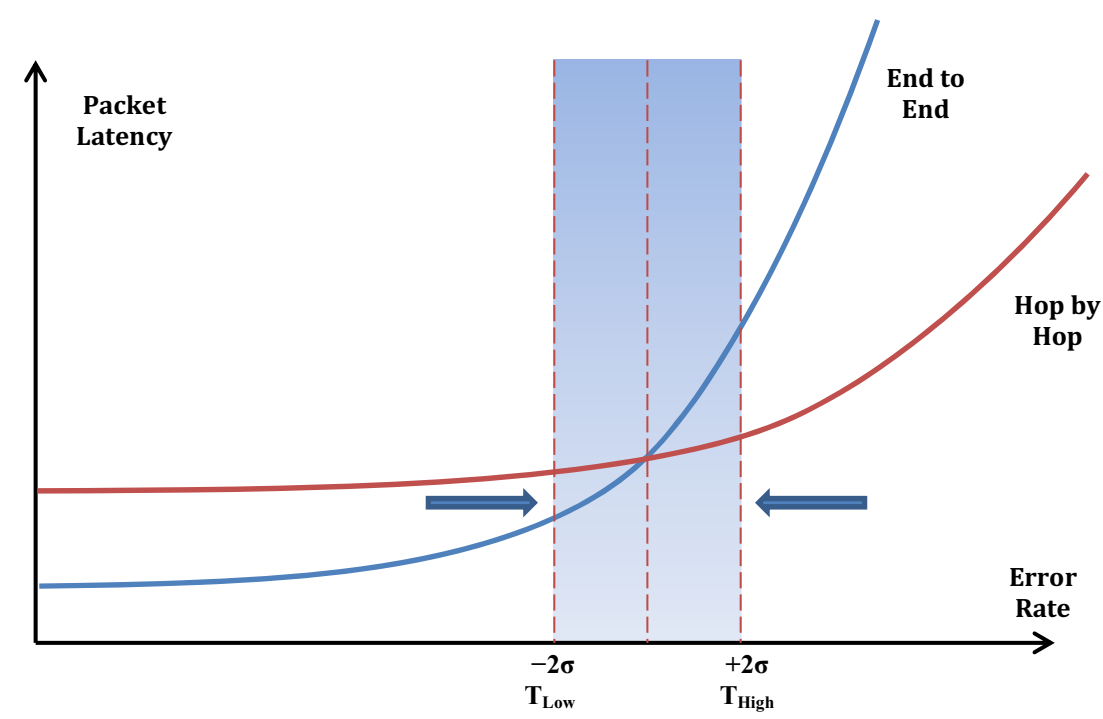

Figure 4. Transition point of end-to-end and hop-by-hop loss recovery methods

Algorithm 1. Pseudo code of the switching process of Loss Recovery Mechanism

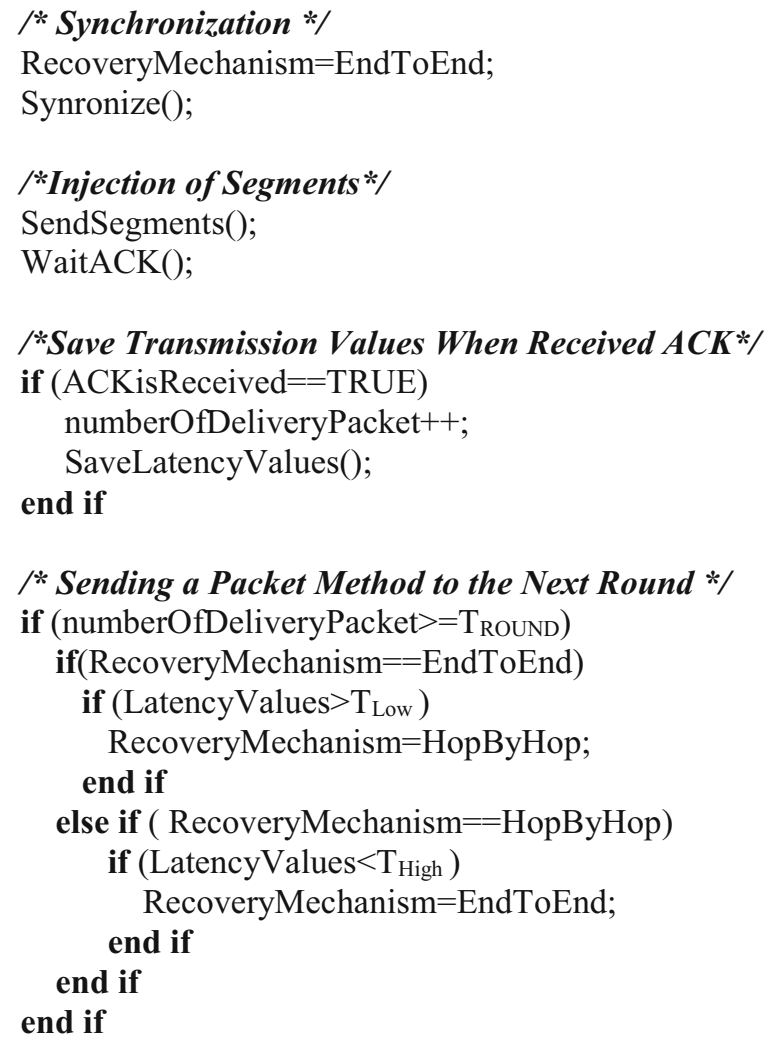

As shown in algorithm 1, synchronization process must be performed between sender and receiver nodes. Afterwards, the source node sends segments and awaits ACK to make sure that all segments have arrived to the target successfully. When ACK packet was received successfully by the source node, the packet round value $T_{\text {Round }}$ is automatically increased and the packet latency values are stored into the source node. If the received packet value is equal to round number, the average latency value of this round is calculated and necessary comparisons are performed. According to the obtained values, the next packet sending method is determined.

The ALORT protocol operates using end-to-end and hop-by-hop LRMs. We designed a simple analytical model of ALORT that operates as hop by hop and end to end. The analytical model of ALORT that operates as hop by hop is shown in Eqs. (1)-(3). Here er represents packet loss rate of environmental conditions.. We assume that $e r$ is constant. The probability of a successful delivery of a packet between two nodes that allows $a$ retransmission is shown as follows.

$$
\begin{gathered}
(1-e r)+\operatorname{erx} \varphi(a) \\
\varphi(a)=\tau(1)+\tau(2)+\tau(3)+\tau(4)+\ldots \tau(a) \\
\tau(a)=((1-e r-\tau(1)-\tau(2)-\tau(3)-\tau(4) \ldots \tau(a-1)
\end{gathered}
$$

$\varphi(a)$ represents the probability of successful recovery of a missing segment and $\tau(a)$ represents the probability of the successful recovery of the missing segment at ath retransmission.

The analytical model of ALORT that operates as end to end is shown in Eq. (4). Here er represents packet loss rate of environmental conditions. We assume that er is constant. The probability of a successful delivery of a packet between two nodes is shown as follows.

$$
\begin{aligned}
\tau(a)= & 1-(1-e r)+(1-e r)^{2}+(1-e r)^{3} \\
& +(1-e r)^{4} \ldots(1-e r)^{a} .
\end{aligned}
$$


Here $\tau(a)$ represents the probability of the successful recovery of the missing segment at ath retransmission.

In end-to-end method, source nodes send a synchronization (SYNC) message to the target node in order to start sending packet as in TCP. It contains some information such as source node ID, destination node ID, total number of segments, ACK number and hop count. The source node that sends SYNC message sets up $T_{\text {senk }}$ timer, although there is corruption possibility of message.

If target node accepts to receive packet, the target node that receives the SYNC message sends a SYN_ACK message to the source node. If SYN_ACK message does not arrive to source node until $T_{\text {senk }}$ timer overflows, the source node decides that the message is corrupted and sends SYNC message to the target again. If source node receives SYN_ACK message successfully, it will send an ACK message to the target node that indicates sending segment starts. Then, source node starts to send segments sequentially without awaiting ACK message. Intermediate nodes that receive segments from source node transmit these segments to the next node without waiting. Classic end-to-end LRM does not provide enough performance in high error rates, because corrupted segments are required from source node and this process takes too long time in multi-hop networks. If end-to-end method will be used, it should be developed. Therefore, end-to-end LRM of proposed ALORT protocol was developed for improving end-to-end performance and it was inspired by e2e TinyTCP [26, 27] protocol.

In proposed end-to-end method, all new segments that arrive to the intermediate nodes are stored in node buffer and are forwarded to the next node without waiting. When segments were corrupted, they are recovered more quickly from intermediate nodes with buffering segments.

Most of the segments do not arrive from source to the target node within the specified time in high channel error rates. Therefore, recovery of missing segments takes too long time. In ALORT protocol, "Middle NACK" architecture is used to speed up recovery process of the missing segments. In Middle NACK architecture, target node tries to recover from intermediate nodes or source node in certain time intervals. According to Middle NACK architecture, target node that receives SYNC_ACK packet sets a timer, namely, $T_{\text {Nack }}$, stated by formula in Eq. (5). Segment ratio is a parameter indicating in which segments the Middle NACK process will start. This parameter is determined by the number of total segments in the packet. According to the simulations, while segment ratio is $10 \%$ of the total number of segments, the optimum packet overhead and packet latency values are obtained. For a packet consisting of 50 segments, timer is set for each five segments' time until that time should reach the target. Segments should arrive to the target node within the predetermined time. In the equation, $T_{\text {Arrive }}$ refers to average arrival time of a segment. $\beta$ is the safety factor and has a value greater than 1 based on channel error rates.

$$
T_{\text {NACK }}=\left(\text { Segment Ratio } \times T_{\text {Arrive }}\right) \beta
$$

When $T_{\text {Nack }}$ timer overflows, target node checks all segments in its buffer and detects all missing segments from the first to the segment number that should arrive to the target node. If there are missing segments, the node generates a NACK message containing ID of missing segments and sends it in the reverse direction. The intermediate nodes that receive NACK message check their own buffers and look at whether missing segments are in their own buffers. Intermediate nodes send segments to the target node located within itself and request segments not within itself from previous node with NACK message. If NACK message was sent by intermediate nodes, recovery process would have ended up at next $T_{\text {Nack }}$ timer overflows. In this case, the recovery process is started again for unrecovered segments in previous cycle. The recovery process continues until last segment range in this way. In the range of last segment, all the missing segments are determined by controlling target node's buffer from beginning to last with overflowing of NACK timer. According to the number of missing segments which were determined, NACK timer is reloaded by formula in Eq. (6). Total Missed Segments show number of segments that did not receive successfully. $T_{\text {Arrive }}$ refers to average latency time of a segment from source to target node and $\beta$ is the safety factor and has a value greater than 1 based on channel error rates. The next installation values of the NACK timer are updated by the number of remaining missing segments.

$$
T_{\mathrm{NACK}}=\left(\text { Total Missed Segments } \times T_{\text {Arrive }}\right) \beta
$$

ALORT protocol also uses hop-by-hop LRM together with end-to-end LRM. The hop-by-hop method of ALORT was inspired by PSFQ [28]. In this method, segments of packet are injected to the network by source node at periodic intervals. Intermediate nodes transmit received segments to the other nodes in a determined time interval. Loss segment detection is determined by segment sequence number. In this method, if a larger sequence number arrives to the node instead of expected sequence number, the node will assume that intermediate segments were corrupted. The node sends a NACK packet containing missing segment numbers to the previous node and waits to receive missing segments. After segments are recovered, they are transmitted to upstream nodes in sequence. In hop-by-hop method, intermediate nodes recover missing segments insistently. So high packet delivery ratio is obtained in hard channel error rates.

In segment transmission process, source node injects segments to the network at periodic intervals. Delay between segments provides to be recovered of the corrupted segments at the intermediate nodes. In recommended hop-by-hop LRM, waiting time between segments is calculated with the help of two timers called $T_{\min }$ and $T_{\max }$. Source node sends segments in sequence to neighbouring nodes in upstream direction within each 
$T_{\text {min }}$ interval. The intermediate node that receives segments checks whether there are received segments in its buffer. If the received segment is not in buffer, in other words, if a new segment is received, the node will store segment and control segment number. If there is no gap in the sequence number of segments, the intermediate node sends segment with a delay randomly determined between $T_{\min }$ and $T_{\max }$. Thus, segments are propagated from source to the target node. If there is a gap in received segment numbers, the node will switch to loss recovery mode and identify missing segments. It tries to recover them from nodes in the reverse direction.

When ALORT operates with hop-by-hop method, it uses unordered segment transmission technique. In PSFQ protocol, if the wrong segment arrives to the node, it will switch to loss recovery mode. It will be held in buffer until the missing segments are recovered. This situation would cause unnecessary latency. To find a solution to this situation, ALORT does not hold the segment that arrives in the wrong order and transmits them to the other nodes. In this case, ALORT sets a bit in the segment called "unordered" to prevent switching to loss recovery mode of upstream nodes unnecessarily. Thus, nodes realise that a segment has arrived with unordered transmission and transmits it to the next node without switching to loss recovery mode. The first intermediate node that segment arrives in the wrong order sets "unordered" bit and transmits it to the next intermediate node. The other intermediate nodes transmit directly to the next node without going into loss recovery mode. In this way, segments arrive to the target node with less latency.

If the arrived segment number is different from expected and "unordered" bit is not set, the intermediate nodes will switch to recovery mode. In the loss recovery mode, to recover missing segments from the previous node is intended in $T_{\text {max }}$ period. Node that switches to loss recovery mode finds missing segments and requests them from neighbour nodes with a NACK packet. Recovering missing segments with a single NACK packet is extremely difficult, especially in hard channel error rates. In fact, it is possible that the NACK packet is corrupted. In order to overcome this disadvantage, ALORT uses a timer named $T_{\mathrm{r}}$, similarly to PSFQ. $T_{\mathrm{r}}$ timer is determined according to the arrived time of missing segments. If all missing segments are recovered without overflowing $T_{\mathrm{r}}$ timer, loss recovery process would be terminated. However, if some of the missing segments are recovered in $T_{\mathrm{r}}$ period, a NACK packet for the remaining missing segments is created and re-sent. Then $T_{\mathrm{r}}$ is established. This process is tried maximum five times in PSFQ. In ALORT, this process is determined adaptively by a number of missing segments. Normally in a loss recovery mode, approximately 12 missing segments can be recovered. Therefore, if the number of missing segments is between 1 and 4, loss recovery process is repeated three times, between 5 and 6 the process is repeated twice, and more than 6 it is repeated once. If the related segments cannot be recovered despite all attempts, the loss recovery process will be cancelled. This is to avoid sending of unnecessary segments and to provide energy conservation.

The missing segments in hop-by-hop method are determined by sequence number of received segments successfully. For example, for a packet consists of six segments, if the target node receives first and second segments and does not receive the third, fourth and fifth segments, the sixth segment must be received successfully in order to detect the missing three segments. So the sixth segment is lost and does not arrive to the target node. Target node cannot detect the missing segments and enters a deadlock. In order to solve these problems mentioned in the example, a periodic timer is usually used. ALORT uses proactive loss recovery mechanism named as $T_{\text {pro }}$. Target node that enters deadlock with $T_{\text {pro }}$ and waits missing segments exits standby mode with overflowing timer and initiates proactive loss recovery process. In proactive mode, target node determines all of missing segments of packet and requests them from nodes in the reverse direction with a NACK. The intermediate node that receives NACK checks whether the missing segments are its own buffer memory. Then the intermediate nodes send segments towards the target and request missing segments from nodes in the reverse direction with a new NACK packet.

The formula used in the first-time installation of $T_{\text {pro }}$ timer is shown in the following equation:

$$
T_{\text {pro }}=\left(S_{\max }-S_{\text {last }}\right) x T_{\max } \times \alpha(\alpha=>1) .
$$

In the equation, $\alpha$ represents a parameter determined with channel error rates and adjusts latency, $S_{\max }$ represents total number of segments, $S_{\text {last }}$ represents last received segment number, $T_{\max }$ represents maximum latency time for transmission of a segment from a node to the other. According to Eq. (7), when $T_{\text {pro }}$ timer overflows, target node checks all missing segments and determines a new load value according to total missing segments. In this way, $T_{\text {pro }}$ timer must be updated again until all missing segments are recovered. The formula that is used to update $T_{\text {pro }}$ timer is shown in the following equation:

$$
T_{\text {pro }}=(\text { Miss Segment Number }) x T_{\max } \times \alpha(\alpha=>1) \text {. }
$$

The hop-by-hop method of ALORT usually works in hard channel error rates. So proactive loss recovery mode is extremely important. However, corruption probability of NACK packets that target node will be sent is highly probable in proactive mode. When NACK packets are corrupted, next overflowing of $T_{\text {pro }}$ timer must be waited for recovery of missing segments. It causes to increase latency significantly. For this reason, the target node sends a new NACK packet in each $T_{\mathrm{r}}$ time. Thus, loss recovery process is guaranteed to be more insistent. It must be maximum three times as described in the previous section as well as in the structure of adaptive loss recovery. 
When ALORT operates with hop-by-hop method, ACK packet is sent to the source node after successful completion of packet transmission. In this way, source node successfully realises packet transmission. In addition, some values in the packet such as packet latency time and total numbers of NACK obtained during packet transmission are transmitted to the target node.

\section{Simulation framework}

The ALORT protocol is applied by using the MIXIM simulation framework. MIXIM is a very powerful framework to simulate wireless and mobile networks by using the discrete event simulator OMNET++. MIXIM has a rich toolbox to support and facilitate the simulation and performance analysis of wireless networks. At the same time, MIXIM allows hiding of the complexity of such simulations and the developer uses the interface easily. This situation facilitates the development and implementation of models and some protocols for WSNs [29].

In this section, tests and experimental results regarding ALORT protocol are presented. The ALORT algorithm is based on adaptive LRMs for WSNs in the literature. The DTC is an end-to-end loss recovery algorithm, which gives efficient results in the fields of energy and packet latency values on low channel error rates in literature. The PSFQ is an algorithm based on hop-by-hop method, which gives efficient results in the field of packet delivery ratio and packet latency on high channel error rates in literature. For these reasons, the ALORT that was designed as an adaptive loss recovery transport algorithm for WSNs is compared to the DTC and PSFQ algorithms according to the number of nodes in terms of energy, packet latency and packet delivery ratio. Also, protocols are compared by changing channel error rates. Packet latency is defined as the time difference between an event that is generated in a source node and its delivery to the sink node. We include lost packets in calculating the latency value. The packet delivery ratio is the ratio of the total number of packets received by sink to the total number of packets generated by source nodes. Energy efficiency is the energy consumed by packet delivery that is delivered to a sink node. The energy consumption, packet latency and packet delivery ratio results are graphically shown.

\subsection{Simulation results and discussion}

In the experiments, we analysed performance of the proposed algorithm ALORT when varying the LRMs of the simulation in different channel error rates. Performance analysis of ALORT protocol was performed with MIXIM framework that runs under OMNET++-based simulation software. We simulated networks with 100 sensor nodes randomly distributed in a $100 \mathrm{~m} \times 100 \mathrm{~m}$ square area. One output (sink) node was arranged to one of the end points of the area. Power capacity, power consumption, radio transmission distances of nodes were selected in accordance with MICAZ node. Transmission rate of nodes was selected 2 Mbit/s. 802.15.4 [30] protocol was used in MAC layer and WiseRoute [31] protocol was used in routing layer. Packet consists of 50 segments and total size is $2.5 \mathrm{~Kb}$. $T_{\max } 60 \mathrm{~ms}, T_{\min } 40 \mathrm{~ms}$ and $T_{\mathrm{r}}$ was selected in hop-by-hop method of ALORT protocol. PSFQ protocol that uses hopby-hop method and DTC [32] protocol that uses end-to-end method were modelled with OMNET ++ environment for comparing performance of the proposed ALORT protocol. Evaluating the performance of algorithm, throughput, packet latency and energy efficiency values of the proposed protocol were observed and compared with the other protocols. In table 1, parameters that used in simulation are listed.

Packet delivery ratio indicates successful delivery of the packets sent from source to the target node. Several simulations were performed for evaluating packet delivery ratio

Table 1. Simulation environment settings.

\begin{tabular}{lccc}
\hline & \multicolumn{3}{c}{ Meaning } \\
\cline { 2 - 4 } Notation & ALORT & PSFQ & 100 \\
\hline Number of nodes $(\mathrm{N})$ & 100 & 100 & $100 \mathrm{~m}$ \\
Transmission range $(\mathrm{r})$ & $100 \mathrm{~m}$ & $100 \mathrm{~m}$ & CSMA802.15.4 \\
MAC protocol & CSMA802.15.4 & CSMA802.15.4 & WiseRoute \\
Routing protocol & WiseRoute & WiseRoute & $2 \mathrm{Mbit} / \mathrm{s}$ \\
Transmission speed & $2 \mathrm{Mbit} / \mathrm{s}$ & $2 \mathrm{Mbit} / \mathrm{s}$ & 50 \\
Total segment number & 50 & 50 & $2.5 \mathrm{~Kb}$ \\
Packet size & $2.5 \mathrm{~Kb}$ & $2.5 \mathrm{~Kb}$ & $0-70 \%$ \\
Bit error rate & $0-70 \%$ & $0-70 \%$ & - \\
$T_{\text {max }}$ & $60 \mathrm{~ms}$ & $100 \mathrm{~ms}$ & - \\
$T_{\text {min }}$ & $40 \mathrm{~ms}$ & $50 \mathrm{~ms}$ & - \\
$T_{\mathrm{r}}$ & Adaptive & Adaptive & - \\
NACK try number & Adaptive & 5 & - \\
\hline
\end{tabular}




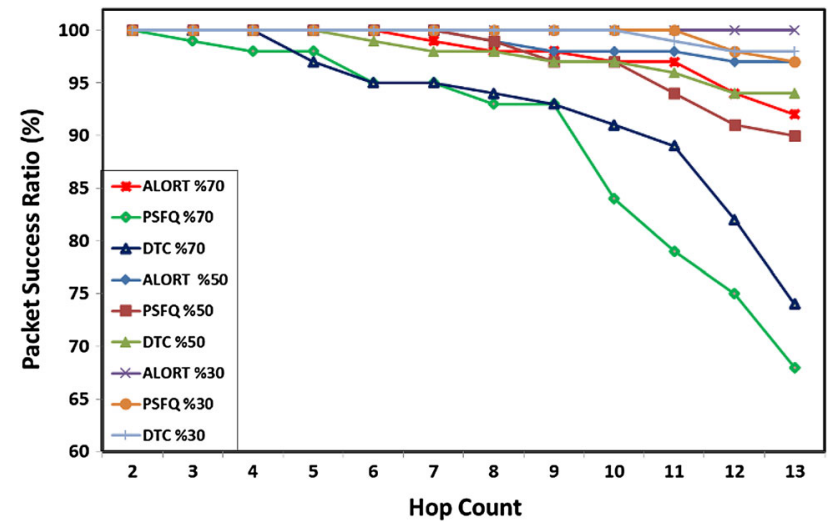

Figure 5. Packet delivery ratios of three protocols in different error rates.

of ALORT protocol and others. Measuring performances of protocols in different channel error rates, packet sending processes were performed as $30 \%, 50 \%$ and $70 \%$ error rates and in different hop numbers (2-13). In the simulations, source nodes sent 100 packets to the target nodes and these packets that are likely to arrive to the target nodes within a specific time were checked.

In figure 5, the average packet delivery ratio of three protocols in different error rates are shown. In 30\% error rate, all protocols can forward all packets to the target successfully especially for low hop counts. If hop number is more than 10, packet delivery ratio of DTC and PSFQ protocols will decrease. However, ALORT protocol performs $100 \%$ packet delivery in $30 \%$ error rate for all hop values. In error rate of 50\%, PSFQ protocol at least $90 \%$ of packets, DTC protocol 94\% of packets and ALORT protocol $97 \%$ of packets are successfully delivered to the target node. When the error rate increases to $70 \%$, performance of PSFQ and DTC protocols significantly reduces. But

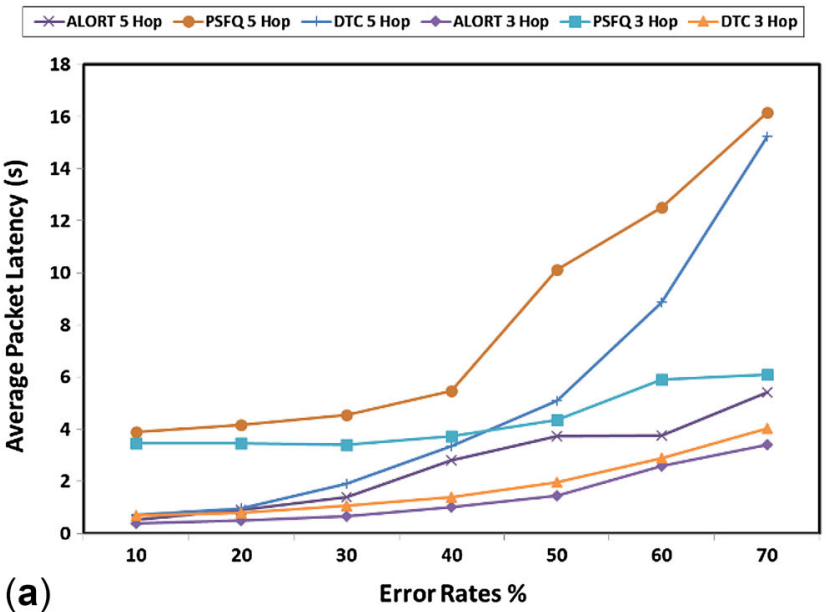
ALORT protocol delivers 92 of 100 packets successfully.

When examining the results, performance of PSFQ and DTC protocols significantly reduces with increasing number of hops. When hop count increases, the recovery process of corrupted packets will take longer time. So packet latency will increase. Therefore, the number of packets that are not arrived to the target node in time increases and packet delivery ratio decreases. As shown in figure 4, ALORT provides better throughput values. It is the most important reason that ALORT protocol uses end-to-end and hop-by-hop methods adaptively according to channel error rates. So packets are delivered to the target node more quickly and with higher packet delivery ratio.

The other aim of a transport layer protocol is to reduce end-to-end packet latency. End-to-end latency is a time value of packet that arrived from source to the target node. It is a very important parameter in delay-sensitive applications. In figure 6, end-to-end latency values of ALORT, DTC and PSFQ protocols in different channel error rates are seen. Examining the figure, ALORT and DTC protocols obtain lower latency values than PSFQ in low channel error rates. However, ALORT obtains lower latency values than PSFQ and DTC in high channel error rates.

We performed analytical tests of ALORT protocol at seven hops network. Figure 7 shows the analytical results of end-to-end latency values of ALORT, DTC and PSFQ protocols. The results are similar to simulation values.

Figure 8 shows the simulation results that is run in random channel error rates for $1200 \mathrm{~s}$. The obtained values are average latency value per packet. Examining the figure, it is seen that end-to-end latency value of PSFQ is much more than ALORT and DTC protocol. If DTC is compared with ALORT protocol, ALORT obtains better values for all hop values. For example, in five hops network, PSFQ, DTC and ALORT protocols deliver a packet to the target node in $6.06 \mathrm{~s}, 2.24 \mathrm{~s}$ and $1.89 \mathrm{~s}$, respectively. Because ALORT protocol uses hop-by-hop LRM in high error rates and insists NACK in proactive loss recovery and proactive

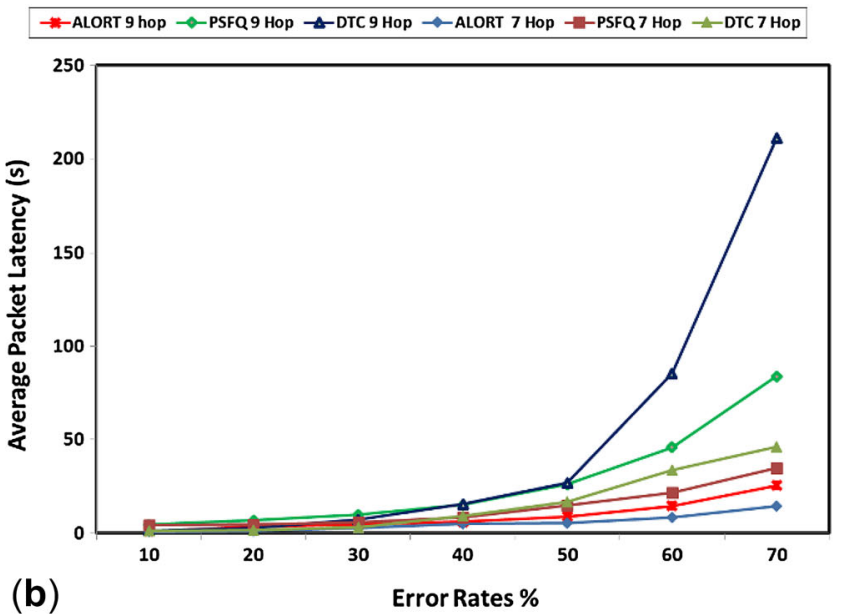

(b)

Figure 6. End-to-end latency values of ALORT, PSFQ and DTC protocols. (a) Three to five hops average packet latency time. (b) Seven to nine hops average packet latency time. 


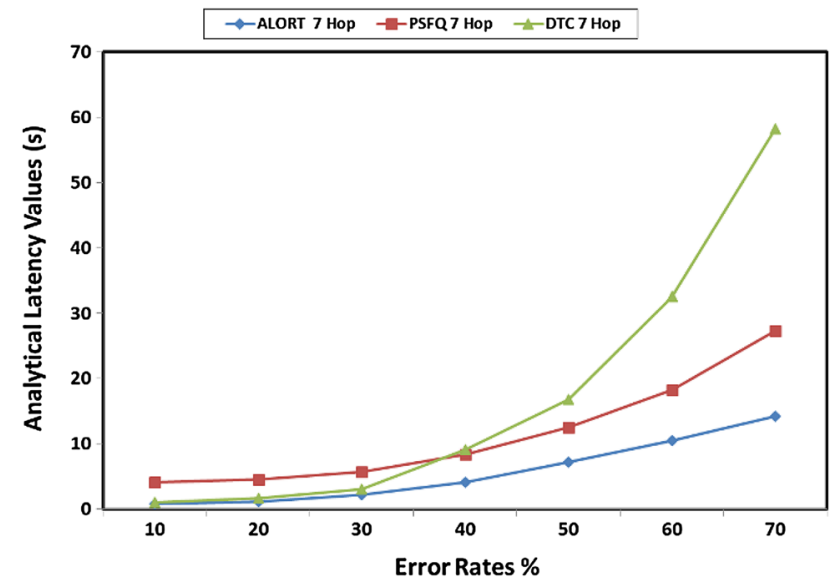

Figure 7. Analytical results of end-to-end latency values of ALORT, DTC and PSFQ.

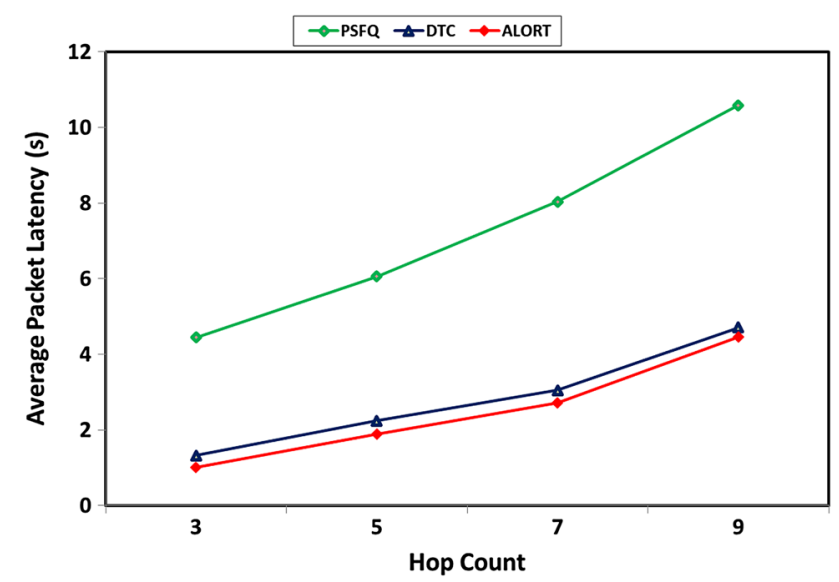

Figure 8. End-to-end latency values of ALORT, PSFQ and DTC protocols in random channel error rates.

NACK and it uses end-to-end method in low error rates and uses Middle NACK architecture. So it provides lower endto-end latency values.

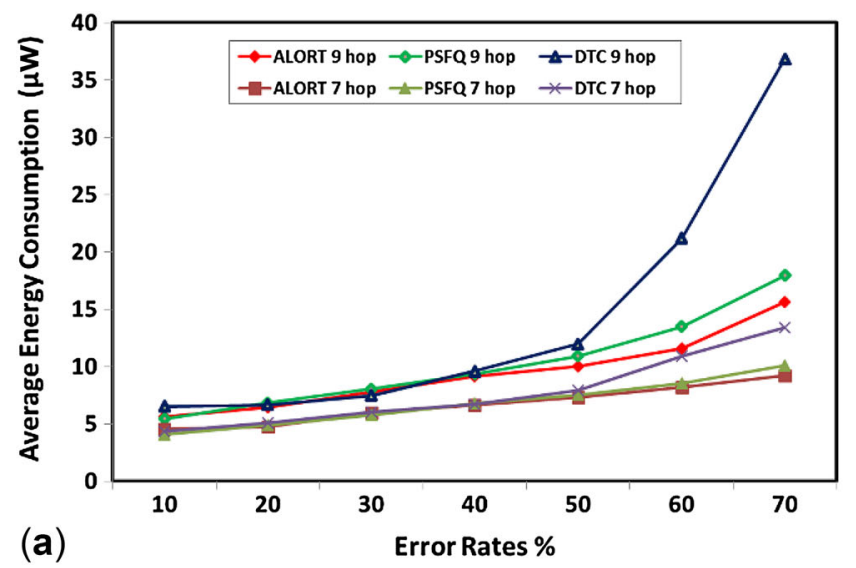

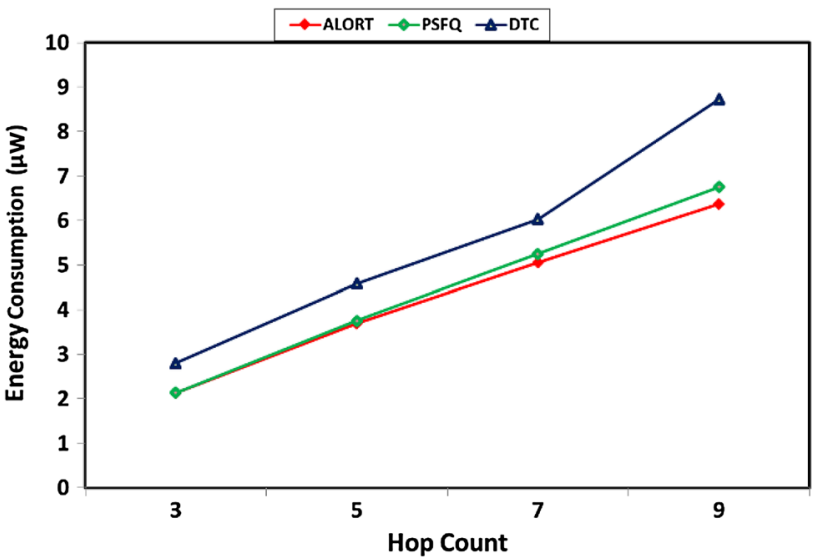

Figure 10. Energy consumption values per packet of ALORT, PSFQ and DTC protocols in random channel error rates.

In this study, energy consumption parameter represents expended total energy of a packet during delivery from source to the target node. This parameter is extremely important especially in applications with low energy sources. In figure 9, average energy consumption value per packet of ALORT, DTC and PSFQ protocols can be seen with different channel error rates. Examining the figure, ALORT and DTC protocols waste a bit more energy than PSFQ in low channel error rates. Therefore, DTC and ALORT protocols send synchronization packets before sending packets. But PSFQ protocol does not use synchronization process. In high error rates, energy consumption of ALORT is better than the other two protocols. DTC protocol has end-to-end method, so packet corruption rate increases excessively in high error rates and, consequently, energy consumption will be much higher.

In figure 10, energy consumption results of simulation that run for $1200 \mathrm{~s}$ in random channel error rates are seen. The obtained values are average energy consumption per packet. Examining the figure, it is seen that energy

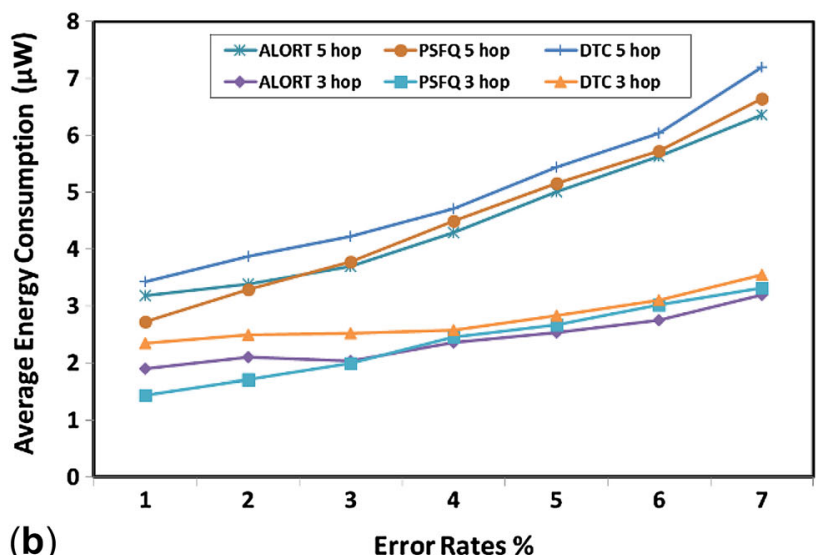

(b)

Figure 9. Average energy consumption of ALORT, PSFQ and DTC. (a) Average energy consumption per packet for seven and nine hops. (b) Average energy consumption per packet for three and five hops. 
consumption value of DTC protocol is much more than ALORT and PSFQ. If ALORT is compared with PSFQ protocol, it can be seen that ALORT protocol obtains very similar energy consumption values in low hop values. ALORT consumes lower energy in high hop values. For example, in nine hops network, DTC 8,72 $\mu \mathrm{W}$, ALORT $6,37 \mu \mathrm{W}$ and PSFQ 3,75 $\mu \mathrm{W}$ consume energy during sending a packet from source to the target node. One of the most important reasons for this situation is that ALORT uses adaptive LRM. Thus, energy conservation is achieved by avoiding unnecessary segment transmission.

\section{Conclusion}

In this study, reliable and delay-sensitive transport layer protocol called ALORT design for WSNs is performed. The most significant difference of ALORT protocol from others in the literature is that it can use both hop-by-hop and end-to-end LRMs adaptively. In this way, ALORT protocol can provide lower latency, lower energy cost and high reliability values in different channel error rates. According to the results of the detailed simulations, ALORT can transmit packets less than $92 \%$ even in the worst channel error rates from end to end. In addition, when ALORT protocol is compared with PSFQ, it is seen that ALORT provides lower latency values of 1:3 ratio according to the PSFQ in many scenarios. When ALORT compared with DTC, this ratio is slightly lower, but especially it increases in high error rates and more hop counts. In terms of energy consumption, ALORT protocol appears to be energy-efficient protocol.

\section{Acknowledgment}

This research was supported by a research grant from by Sakarya University BAPK project numbered 2010-05-02008 (a new transport protocol design for wireless sensor networks).

\section{References}

[1] Chonggang W, Sohraby K, Bo L, Daneshmand M and Yueming H 2006 A survey of transport protocols for wireless sensor networks. IEEE Netw. Mag. 20(3): 34-40

[2] Dinusha R A J and Vidyasagar M P 2013 Wireless sensor network transport protocol: a critical review. J. Netw. Comput. Appl. 36(1): 134-146

[3] Pereira P, Grilo A, Rocha F, Nunes M S, Casaca A, Chaudet C, Almström P and Johansson M 2007 End-to-end reliability in wireless sensor networks: survey and research challenges EuroFGI Workshop on IP QoS and Traffic Control, Lisbon, Portugal

[4] Kaushal K, Kaur T and Kaur J 2015 A survey on reliable and congestion control transport protocols of WSN. Int. J. Comput. Appl. 110(7): 32-35
[5] Iyer Y G, Gandham S and Venkatesan S 2005 STCP: a generic transport layer protocol for wireless sensor networks. In: Proceedings of IEEE ICCCN, San Diego, CA, USA, pp. 449-454

[6] Sankarasubramaniam Y, Akan O B and Akyildiz I F 2003 ESRT: event-to-sink reliable transport in wireless sensor networks. In: Proceedings of fourth ACM international symposium on mobile ad hoc networking and computing, New York, NY, USA, pp. 177-188

[7] Shaikh F K, Khelil A, Ali A and Suri N 2010 TRCCIT: tunable reliability with congestion control for information transport in wireless sensor networks. In: Proceedings of the International Wireless Internet Conference (WICON), Singapore, pp. 1-9

[8] Alam M and Hong C 2009 CRRT: congestion-aware and rate-controlled reliable transport in wireless sensor networks. IEICE Trans. Commun. E92(B): 184-189

[9] Gungor V C and Akan Ö B 2008 A real-time and reliable transport $(\mathrm{RT})^{2}$ protocol for wireless sensor and actor networks. IEEE/ACM Trans. Netw. 16(2): 359-370

[10] Tezcan N and Wang W 2007 ART: an asymmetric and reliable transport mechanism for wireless sensor networks. Int. J. Sensor Netw. 2(3/4): 188-200

[11] Paek J and Govindan R 2007 RCRT: rate controlled reliable transport for wireless sensor networks. In: Proceedings of the 5th international conference on embedded networked sensor systems, Sydney, Australia, pp. 305-319

[12] Kim S, Fonseca R, Dutta P, Tavakoli A, Culler D and Levis P 2007 Flush: a reliable bulk transport protocol for multihop wireless networks. In: Proceedings of the 5th international conference on Embedded networked sensor systems, Sydney, Australia, pp. 351-365

[13] Zhou Y and Lyu M 2005 PORT: a price-oriented reliable transport protocol for wireless sensor network. In: Proceedings of 16th IEEE international symposium on software reliability engineering, Chicago, pp. 117-126

[14] Hull B, Jamieson K and Balakrishnan H 2004 Mitigating congestion in wireless sensor networks. In: Proceedings of ACM Sensys 04, Baltimore, MD, USA

[15] Wan C Y, Eisenman S B and Campbell A T 2003 CODA: congestion detection and avoidance in sensor networks. In: Proceedings of the 1st International Conference on Embedded Networked Sensor Systems (SenSys'03), Los Angeles, CA, USA, pp. 266-279

[16] Ee C T and Bajcsy R 2004 Congestion control and fairness for many-to-one routing in sensor networks. In: Proceedings of ACM Sensys 04, Baltimore, MD, USA, pp. 148-161

[17] Wang C, Sohraby K, Lawrence V, Li B and Hu Y 2006 Priority-based congestion control in wireless sensor networks. In: Proceedings of IEEE International Conference on Sensor Networks, Ubiquitous, and Trustworthy Computing, pp. 22-31

[18] Woo A and Culler D C 2001 A transmission control scheme for media access in sensor networks. In: Proceedings of 2001 ACM Annual International Conference on Mobile Computing and Networking (Mobicom 01), Rome, Italy, pp. 221-235

[19] Wan C Y, Eisenman S B, Campbell A T and Crowcroft J 2005 Siphon: over-load traffic management using multi-radio virtual sinks in sensor networks. In: Proceedings of ACM SenSys 05, San Diego, California, USA, pp. 116-129 
[20] Levis P, Patel N, Culler D and Shenker S 2004 Trickle: a self-regulating algorithm for code propagation and maintenance in wireless sensor networks. In: Proceedings of First Symposium Networked Sys. Design and Implementation (NSDI), pp. 15-28

[21] Monowar M M, Rahman M O, Pathan A K and Hong C S 2008 Congestion control protocol for wireless sensor networks handling prioritized heterogeneous traffic. In: Proceedings of the 5th Annual International Conference on Mobile and Ubiquitous Systems Computing Networking and Services, Brussels, Belgium

[22] Wang C, Sohraby K and Li B 2005 SenTCP: a hop-by-hop congestion control protocol for wireless sensor networks. In: Proc. IEEE INFOCOM, Miami, USA

[23] Kiraz A and Çakıroğlu M 2014 A survey of congestion control protocols providing energy conservation in wireless sensor networks. Turkish J. Eng. Sci. Technol. 2(1): 12-22

[24] Heimlicher S, Nuggehalli P and May M 2007 End-to-end vs. hop-by-hop transport. ACM SIGMETRICS Perform Eval Rev, 35(3): 59-60

[25] Wessel K, Swigulski M, Köpke A and Willkomm D 2009 MiXiM - the physical layer an architecture overview. In: Proceeding of the 2nd International Workshop on OMNET++, Rome, Italy

[26] Dunkels A, Alonso J and Voight T 2004 Making TCPIP viable for wireless sensor networks. In: European workshop on wireless sensor networks (EWSN), Germany
[27] Dunkels A, Voigt A T, Alonso J, Ritter H and Schiller J 2004 Connecting Wireless SensorNets with TCPIP networks. In: Proc. Second International Conference on Wired Wireless Internet Communications (WWIC2004), vol. 2957, pp. $143-152$

[28] Wan C Y, Campbell A T and Krishnamurthy L 2002 PSFQ: a reliable transport protocol for wireless sensor networks. In: Proceedings of ACM International Workshop on Wireless Sensor Networks and Applications, Atlanta, GA, USA, pp. $1-11$

[29] Köpke A, Swigulski M, Wessel K, Willkomm D, Klein H P T, Parker T E V, Visser O W, Lichte H S and Valentin S 2008 Simulating wireless and mobile networks in OMNET++ the MiXiM vision. In: Proceeding of the First International Workshop on OMNeT++, France

[30] Dopico N I, Gil-Soriano C, Arrazola I and Zazo S 2010 Analysis of IEEE 802.15.4 Throughput in Beaconless Mode on micaZ under TinyOS. In: Vehicular Technology Conference Fall (VTC 2010-Fall), 2010 IEEE 72nd, pp. 1-5

[31] Piguet D, Rousselot J, Dallemagne P and Kassapoglou-Faist C 2009 Routing for mobile wireless sensor networks. Scientific and Technical Report

[32] Dunkels A, Voigt T, Ritter H and Alonso J 2004 Distributed TCP caching for wireless sensor networks. In: Proceedings of the 3rd Annual Mediterranean AdHoc Networking workshop, Turkey 